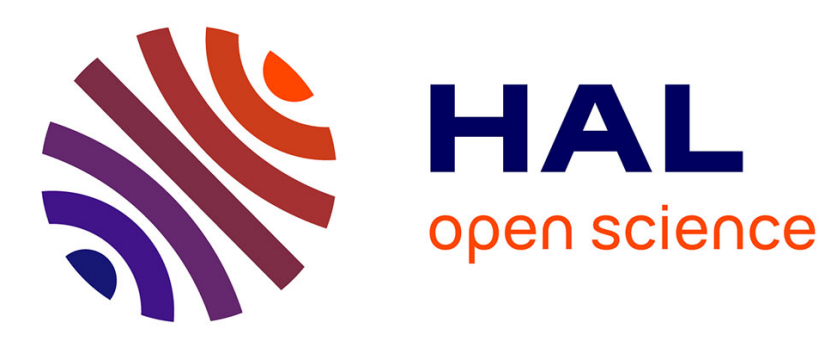

\title{
Robust stabilization of an airlaunch system after launching phase
}

\author{
Cuong Nguyen Van, Riccardo Marino, Gilney Damm
}

\section{To cite this version:}

Cuong Nguyen Van, Riccardo Marino, Gilney Damm. Robust stabilization of an airlaunch system after launching phase. 51st IEEE Conference on Decision and Control (CDC 2012), Dec 2012, Maui, HI, United States. pp.3564-3569, 10.1109/CDC.2012.6426432 . hal-00786859

\section{HAL Id: hal-00786859 \\ https://hal.science/hal-00786859}

Submitted on 30 Mar 2021

HAL is a multi-disciplinary open access archive for the deposit and dissemination of scientific research documents, whether they are published or not. The documents may come from teaching and research institutions in France or abroad, or from public or private research centers.
L'archive ouverte pluridisciplinaire HAL, est destinée au dépôt et à la diffusion de documents scientifiques de niveau recherche, publiés ou non, émanant des établissements d'enseignement et de recherche français ou étrangers, des laboratoires publics ou privés. 


\title{
Robust Stabilization of an Airlaunch System after Launching Phase*
}

\author{
Van Cuong Nguyen and Riccardo Marino and Gilney Damm
}

\begin{abstract}
A Multiple Input Multiple Output (MIMO) controller based on the dynamic feedback linearization technique is designed for the robust global stabilization of a new satellite launching strategy called (unmanned) airlaunch. This strategy consists in using a two-stages launching system. The first stage is composed of an airplane (manned or unmanned) that carries a rocket launcher which constitute the subsequent stages. The control objective is to stabilize the aircraft in the launch phase. It is developed and is applied to the full multi-input multioutput model of the aircraft. The considered model is highly nonlinear, mostly as a consequence of possible large angle of attack, sideslip and roll angle. Finally, the present work illustrates through simulations the good performance of the proposed control algorithm.
\end{abstract}

\section{INTRODUCTION}

This work presents the design of a Multiple Input Multiple Output controller for the robust stabilization of a new satellite launching strategy called (unmanned) airlaunch. This strategy consists in using a two-stages launching system. The first stage is composed of an airplane that carries a rocket launcher which constitutes the subsequent stage. The aircraft brings the rocket to a desired drop area, consequently avoiding many costs and risks related to land rocket launching. On the other hand, this procedure brings up many other difficulties connected to the instant of releasing the rocket.

Currently, several airlaunch systems are under development (see [20] and [7]). Most current airlaunch projects use standard or lightly modified airplanes as first stage. For example, there has been tests using F15, C17, B52, L-1011 in Rascal, QuickReach, Proteus and Pegasus projects. Unlike those, other projects aim to develop an airlaunch system that uses an unmanned aerial vehicle (UAV) instead of a standard aircraft with a human pilot inboard. The objective is then to use an UAV to fly the launcher to the desired drop point. There are many advantages in doing so, in first place safety since no human lives are involved during the delicate launching phase. In addition, since there is no need for life supporting devices, weight is restricted to the strict minimum. Finally, mission may take as long as necessary without human restrictions as tiredness.

The present paper addresses the stabilization of the drop phase. It intends to introduce a robust control scheme for this

\footnotetext{
*The research leading to these results has received funding from the European Union Seventh Framework Programme [FP7/2007-2013] under grant agreement n257462 HYCON2 Network of excellence

Van Cuong Nguyen is with IBISC - Université d'Evry Val d'Essonne, Evry, France vancuong.nguyen@ibisc.fr

Riccardo Marino is with "Tor Vergata" University, Electronic Engineering Department, Via del Politecnico, 00133 Rome, Italy marino@ing.uniroma2. it

Gilney Damm is with IBISC - Université d'Evry Val d'Essonne, Evry, France gilney. dammeibisc.fr
}

complex procedure. In fact, airlaunch may be very delicate for many reasons. For example, since the rocket may be almost as heavy as the UAV, this means that the aircraft will instantaneously lose almost half of its mass. The proposed system must replace the human pilot in this stabilization task, with a much more adverse mass ratio. In the same way, the two-stages system is strongly nonlinear and can even for small perturbations be brought quite far from the initial equilibrium point.

Our previous works (see [16] and [15]) based on MIMO conditional integrator developed in a series of papers ([5] and [12]) gave good results on the stabilization of the airlaunch system. However the problem of stabilization of a complete airlaunch system is still not solved. In previous results, at least one of its states remains uncontrolled. On the other hand the thrust force did not play a serious role in the stabilization procedure. As a consequence, the available inputs are being underused while some states still need to be controlled. Finally, the control surfaces become easily saturated.

For all these reasons we suggest a possibility of applying a nonlinear controller for this problem. In [10], a nonlinear flight control, based on inverse dynamics is presented and is improved as a probabilistic robust control of nonlinear uncertain flight system in [21]. The inverse dynamics control provided high performance for large angle of attack conditions. Other nonlinear approaches were based on backstepping control laws (see [18]) and backstepping and neural network control (see [11]) in the case of extreme flight conditions.

A slightly different nonlinear approach from inverse dynamic is presented in [13] for the V/TOL aircraft and for a helicopter in [6] by using nonlinear feedback linearization. In order to be feedback linearizable, the system must satisfy necessary and sufficient conditions shown in [8] and [17]. The regulator is designed, following a system transformation, such that the system tracks the output of a reference model.

Based on the dynamic feedback linearization theory (see [1], [2] and [3]) it was proved that, given some assumptions, a simplified version of the considered system can be dynamic feedback linearizable using a first order integration of the thrust input.

These results have pointed some specific system characteristics that are further exploited in the present work. Here it is used the dynamic feedback control strategy based on the nonlinear model to stabilize the full $12^{\text {th }}$ order airlaunch system in extreme situations after launch phase.

The paper is organized as follows: in section II, we describe the system model. The control design literature is discussed in section III, and its application to the full non- 
linear system model in section IV. The paper is completed by some computer simulations and conclusions.

\section{SySTEM MODEL}

Drop phase is delicate to model, and requires a large amount of data and previous knowledge about the real system. It can also be represented as a hybrid system composed by two (or three) continuous models that are switched. These models represent the system before, (possible during) and after the separation phase. In the present work we have adopted this strategy, we have considered three phases, using two aircraft models.

1) before the separation $\Rightarrow$ the first model is considered at an stable operating condition

2) during the separation $\Rightarrow$ the launch phase itself happens during an interval $T_{i n t}$. During this interval the second model is used, but disturbed by constant aerodynamic force and moment representing an imperfect launch of the rocket from the aircraft

3) after the separation $\Rightarrow$ the disturbances stop, and the second model continues to be used

In order to make our study as much general as possible, the first model is taken as an F-16 with twice its normal mass, while the second model is taken as the complete F16 model. It is important to remark that the first model, in practice, is only used to compute the initial conditions. for the separation phase.

It can be shown that the effect of launching the rocket from the carrier aircraft disturb mostly lift force and pitch and roll moments. We suppose that these disturbing force and moments are constant during the interval $T_{\text {int }}$. We call $F_{z_{p}}, L_{p}$ and $M_{p}$ the disturbances on the lift force, on the roll moment and pitch moment respectively.

We suppose that:

- the perturbation on lift force during $T_{i n t}$ is equal to the air launch vehicle's mass, that means $F_{z}=m g$.

- the perturbation on pitch moment during $T_{i n t}$ is an worst case that is represented by the rocket that remains attached to the aircraft by only one end during $T_{\text {int }}$, applying a rotational movement to the aircraft, so a moment with value $F_{z}=m g l_{r} / 2$ where $l_{r}$ is the rocket length.

- the perturbation on roll moment during $T_{\text {int }}$ is small because of the rocket shape (long and thin).

- the model following the launch phase is the F-16 model. Its initial condition is the state at an equilibrium point of the model previous the launch phase that is the F-16 model but with twice its standard mass.

Following this procedure, the aircraft in the instant following the dynamic airlaunch is described in the body fixed axes as in (1) (see [9] and [19]).

In (1), $I_{x x}, I_{y y}, I_{z z}, I_{x z}$ are the moments of inertia in $\mathrm{kgm}^{2}, \mathrm{~m}$ is the mass of the system $(\mathrm{kg})$ and $g$ the gravity constant. $x, y, z, u, v, w, p, q, r, \phi, \theta, \psi$ are the state variables of the airlaunch aircraft model, they represent three positions, longitudinal velocity, lateral velocity, normal velocity, roll rate, pitch rate, yaw rate, roll angle, pitch angle and yaw angle respectively. $\phi, \theta, \psi$ are expressed in $\mathrm{rad}, p, q, r$ in $\mathrm{rad} / \mathrm{s} . F_{u}, F_{v}, F_{w}$ and $L, M, N$ are aerodynamic forces and moments respectively. These aerodynamic forces and moments are function of all the considered states. In this model, these aerodynamic forces and moments are under look-up table from wind tunnel data measurements. We also introduce $\alpha, \beta, V$ that are angle of attack (rad), sideslip angle $(\mathrm{rad})$ and airspeed $(\mathrm{m} / \mathrm{s})$ in relationship with $u, v, w$. Finally $T$ is the thrust force, the control inputs are respectively aileron $\left(\delta_{a}\right)$, rudder $\left(\delta_{r}\right)$ and elevator $\left(\delta_{e}\right)$ values.

$$
\left\{\begin{array}{l}
\dot{x}=u c \psi c \theta+v(c \psi s \theta s \phi-s \psi c \phi)+w(c \psi s \theta c \phi+s \psi s \phi) \\
\dot{y}=u s \psi c \theta+v(s \psi s \theta s \phi+c \psi c \phi)+w(s \psi s \theta c \phi-c \psi s \phi) \\
\dot{z}=-u s \theta+v c \theta s \phi+w c \theta c \phi \\
\dot{u}=r v-q w-g s \theta+\frac{1}{m}\left(F_{u}+T\right) \\
\dot{v}=p w-r u+g s \phi c \theta+\frac{1}{m} F_{v} \\
\dot{w}=q u-p v+g c \phi c \theta+\frac{1}{m} F_{w} \\
\dot{\phi}=p+\tan \theta(q s \phi+r c \phi) \\
\dot{\theta}=q c \phi-r s \phi \\
\dot{\psi}=\frac{q s \phi+r c \phi}{c \theta} \\
\dot{p}=\frac{1}{I_{x x} I_{z z}-I_{x z}^{2}}\left[\left(I_{y y} I_{z z}-I_{z z}^{2}-I_{x z}^{2}\right) r q-I_{x z}\left(I_{x x}\right.\right. \\
\left.\left.+I_{z z}-I_{y y}\right) p q+I_{z z} L-I_{x z} N\right] \\
\dot{q}=\frac{1}{I_{y y}}\left[\left(I_{z z}-I_{x x}\right) p r+I_{x z}\left(p^{2}-r^{2}\right)+M\right] \\
\dot{r}=\frac{1}{I_{x x} I_{z z}-I_{x z}^{2}}\left[\left(-I_{x x} I_{y y}+I_{z z}^{2}+I_{x z}^{2}\right) p q+I_{x z}\left(I_{x x}\right.\right. \\
\left.\left.+I_{z z}-I_{y y}\right) r q+I_{x x} N-I_{x z} L\right]
\end{array}\right.
$$

where $c$ signifies cosinus and $s$ signifies sinus, e.g. $c \psi=$ $\cos \psi$ and $s \psi=\sin \psi$.

This model is based on wind tunnel data from NASA found in [14], considering the following conditions:

- angle of attack is in the range of $\left[-10^{\circ}, 45^{\circ}\right]$ and sideslip of $\left[-30^{\circ}, 30^{\circ}\right]$

- flag deflection is ignored

- physical constraints for aileron $\left(\left|\delta_{a}\right| \leq 21.5^{\circ}\right)$, rudders $\left(\left|\delta_{r}\right| \leq 30^{\circ}\right)$ and elevator $\left(\left|\delta_{e}\right| \leq 25^{\circ}\right)$

- all actuators are modeled as a first order model with limit rates $60^{\circ} / \mathrm{s}$ for aileron and elevator, and $120^{\circ} / \mathrm{s}$ for rudder.

In particular we use the low quality mode of the F-16 model, and in simulations the aerodynamic data is interpolated and extrapolated linearly from the tables.

Before entering into the design of a Nonlinear Dynamic Feedback Linearization controller for globally stabilizing the airlaunch system after dropping phase, we state the following assumption.

Assumption 1: The control surfaces deflection and angular rates have no effects on the aerodynamic force components $\left(F_{u}, F_{v}\right.$ and $\left.F_{w}\right)$ but only on moments. The components $F_{w}$, $F_{u}$ and $F_{v}$ depend then only on the linear velocities $u, v, w$.

\section{CONTROL DESIGN}

Previous works ([1]), ([2] and [3]) in dynamic feedback linearization theory have proven that the system composed by the first nine differential equations in (1) can be dynamic feedback linearizable using $(p, q, r)$ as control variables, as well as a first order integrator applied on the thrust. We follow and make a step forward on this technique to demonstrate the dynamic feedback linearizability of the complete $12^{\text {th }}$ order flight dynamics. We will use a second order integration of thrust input and develop a nonlinear control algorithm in order to stabilize the aircraft and track a given trajectory.

Because $(p, q, r)$ in (1) can be controlled by $\left(\delta_{a}, \delta_{e}, \delta_{r}\right)$ contained in $(L, M, N)$, we can simplify the angular motion 
of the last three equations in (1) as ${ }^{1}$ :

$$
\dot{p}=\dot{p}_{0} ; \dot{q}=\dot{q}_{0} ; \dot{r}=\dot{r}_{0}
$$

where $\dot{p}_{0}, \dot{q}_{0}, \dot{r}_{0}$ are control inputs.

System (1) is then of the type:

$$
\dot{\xi}_{s}=f_{s}\left(\xi_{s}\right)+\dot{p}_{0} g_{s 1}\left(\xi_{s}\right)+\dot{q}_{0} g_{s 2}\left(\xi_{s}\right)+\dot{r}_{0} g_{s 3}\left(\xi_{s}\right)+\eta g_{s 4}\left(\xi_{s}\right)
$$

where $f_{s}, g_{s 1}, g_{s 2}, g_{s 3}, g_{s 4}$ are obtained from (2) respectively and $\eta=T / m$,

$$
\begin{aligned}
& g_{s 1}=(0,0,0,0,0,0,0,0,0,1,0,0)^{T} \\
& g_{s 2}=(0,0,0,0,0,0,0,0,0,0,1,0)^{T} \\
& g_{s 3}=(0,0,0,0,0,0,0,0,0,0,0,1)^{T} \\
& g_{s 4}=(0,0,0,1,0,0,0,0,0,0,0,0)^{T}
\end{aligned}
$$

We can compute the Lie brackets $a d_{g_{s i}} g_{s j}$ for $1 \leq i, j \leq 4$ and check that

- $\operatorname{span}\left(g_{s 1}, g_{s 2}, g_{s 3}, g_{s 4}\right)$ is involutive

- $\operatorname{span}\left(g_{s 1}, g_{s 2}, g_{s 3}, g_{s 4}, a d_{f} g_{s 1}, a d_{f} g_{s 2}, a d_{f} g_{s 3}, a d_{f} g_{s 4}\right)$ is not involutive

This result implies (see [17]) that system (1) is not static feedback linearizable.

Since $g_{s 4}$, which lies in the direction of thrust, plays an important role in the dynamics of the aircraft, we choose to apply a second order integrator on the thrust $(\eta, \dot{\eta}$ and $\ddot{\eta})$, and to check the condition for dynamic feedback linearization with the augmented system

$$
\left\{\begin{array}{l}
\Delta_{0}=\operatorname{span}\left(g_{s 1}, g_{s 2}, g_{s 3}\right) \\
\Delta_{1}=\Delta_{0}+a d_{f_{s}} \Delta_{0}+\operatorname{span}\left\{g_{s 4}\right\} \\
\Delta_{2}=\Delta_{1}+a d_{f_{s}} \Delta_{1}+\operatorname{span}\left\{g_{s 4}\right\} \\
\Delta_{3}=\Delta_{2}+a d_{f_{s}} \Delta_{2}=\mathbb{R}^{12 \times 1}
\end{array}\right.
$$

System (4) satisfies all sufficient conditions of the theory presented in [3] and [4]. The extended system in (1) with the simplified equations (2) and the second order integrator on thrust is dynamic feedback linearizable. This can be physically explained by the fact that engine dynamics of thrust are of second order type, as mentioned in [10].

The work in [4] has shown that the system composed by the nine first differential equations of (1), using $(p, q, r, \dot{\eta})$ as control variables, can be dynamically feedback linearized. There it is first defined $\zeta_{1}=$ $x, \zeta_{2}=y, \zeta_{3}=z, \sigma_{1}=\eta$. Then, a change of coordinates from $\tilde{X}=(x, y, z, u, v, w, \phi, \theta, \psi, \eta)$ to $\tilde{\zeta}=\left(x, L_{\tilde{f}} x, L_{\tilde{f}}^{2} x, y, L_{\tilde{f}} y, L_{\tilde{f}}^{2} y, z, L_{\tilde{f}} z, L_{\tilde{f}}^{2} z, \sigma_{1}\right)$ makes the nine first differential equations in (1) feedback linearizable in respect to the control variables $(p, q, r, \dot{\eta})$. There, $\tilde{f}$ is defined when the nine first differential equations in (1) are rewritten in the form $\dot{\tilde{X}}=\tilde{f}+\tilde{g} \tilde{u}$.

Distinctly from [4], we will define $\sigma_{1}$ as another variable to avoid singularity of the matrix $\gamma_{1}(\cdot)$ that we will introduce later. For this reason, now the nine first differential equations and the first order integrator on the thrust can be transformed into a new feedback linearizable system as:

$$
\left\{\begin{array}{l}
\dot{\zeta}_{1}=\zeta_{4}=L_{\tilde{f}} x \\
\dot{\zeta}_{2}=\zeta_{5}=L_{\tilde{f}} y \\
\dot{\zeta}_{3}=\zeta_{6}=L_{\tilde{f}} z \\
\dot{\zeta}_{4}=\zeta_{7}=L_{\tilde{f}}^{2} x \\
\dot{\zeta}_{5}=\zeta_{8}=L_{\tilde{f}}^{2} y \\
\dot{\zeta}_{6}=\zeta_{9}=L_{\tilde{f}}^{2} z \\
\dot{\zeta}_{7}=D_{0}^{1}+D_{1}^{1} p+D_{1}^{2} q+D_{1}^{3} r+D_{1}^{4} v_{4} \\
\dot{\zeta}_{8}=D_{0}^{2}+D_{2}^{1} p+D_{2}^{2} q+D_{2}^{3} r+D_{2}^{4} v_{4} \\
\dot{\zeta}_{9}=D_{0}^{3}+D_{3}^{1} p+D_{3}^{2} q+D_{3}^{3} r+D_{3}^{4} v_{4} \\
\dot{\sigma}_{1}=D_{0}^{4}+D_{4}^{1} p+D_{4}^{2} q+D_{4}^{3} r+D_{4}^{4} v_{4}
\end{array}\right.
$$

\footnotetext{
${ }^{1}$ Remark that the three last equations in 1 are linear independent
}

where $v_{4}=\dot{\eta}$ and $D_{j}^{i}$ for $i=1 . .4, j=1 . .3$ are function of $\tilde{X}$ and can be easily computed (see [4]) and

$$
\left\{\begin{aligned}
\zeta_{4}= & u c \theta c \psi+v(c \psi s \theta s \phi-c \phi s \psi)+w(s \theta c \phi c \psi+s \phi s \psi) \\
\zeta_{5}= & u c \theta s \psi+v(s \psi s \theta s \phi+c \phi c \psi)+w(s \theta c \phi s \psi-s \phi c \psi) \\
\zeta_{6}= & -s \theta u+v c \theta s \phi+w c \theta c \phi \\
\zeta_{7}= & c \theta c \psi\left(-g s \theta+F_{u}+\eta\right)+(c \psi s \theta s \phi-c \phi s \psi)\left(g c \theta s \phi+F_{v}\right) \\
& +(s \theta c \phi c \psi+s \phi s \psi)\left(g c \phi c \theta+F_{w}\right) \\
\zeta_{8}= & c \theta s \psi\left(-g s \theta+F_{u}+\eta\right)+(s \psi s \theta s \phi+c \phi c \psi)\left(g c \theta s \phi+F_{v}\right) \\
& +(s \theta c \phi s \psi-s \phi c \psi)\left(g c \phi c \theta+F_{w}\right) \\
\zeta_{9}= & -s \theta\left(-g s \theta+F_{u}+\eta\right) \\
& +c \theta s \phi\left(g c \theta s \phi+F_{v}\right)+c \theta c \phi\left(g c \phi c \theta+F_{w}\right)
\end{aligned}\right.
$$

From (5), we define four new variables $\zeta_{10}=\dot{\zeta}_{7}, \zeta_{11}=\dot{\zeta}_{8}$, $\zeta_{12}=\dot{\zeta}_{9}$ and $\sigma_{2}=\dot{\sigma}_{1}$.

$$
\left[\begin{array}{l}
\zeta_{10} \\
\zeta_{11} \\
\zeta_{12} \\
\sigma_{2}
\end{array}\right]=\chi_{1}(\tilde{X})+\gamma_{1}(\tilde{X})\left[\begin{array}{c}
p \\
q \\
r \\
v_{4}
\end{array}\right]
$$

where

$$
\chi_{1}(\tilde{X})=\left[\begin{array}{c}
D_{0}^{1} \\
D_{0}^{2} \\
D_{0}^{3} \\
D_{0}^{4}
\end{array}\right] ; \gamma_{1}(\cdot)=\left[\begin{array}{cccc}
D_{1}^{1} & D_{1}^{2} & D_{1}^{3} & D_{1}^{4} \\
D_{2}^{1} & D_{2}^{2} & D_{2}^{3} & D_{2}^{4} \\
D_{3}^{1} & D_{3}^{2} & D_{3}^{3} & D_{3}^{4} \\
D_{4}^{1} & D_{4}^{2} & D_{4}^{3} & D_{4}^{4}
\end{array}\right]
$$

$D_{4}^{i}$ for $i=1 . .4$ are function of $\tilde{X}$ and depend on the choice of variable $\sigma_{1}$ to avoid singularity of $\gamma_{1}(\cdot)$.

The angular dynamics of (1) can be rewritten as:

$$
\left[\begin{array}{c}
\dot{p} \\
\dot{q} \\
\dot{r}
\end{array}\right]=\chi_{r}(u, v, w, p, q, r)+\gamma_{r}(u, v, w)\left[\begin{array}{c}
\delta_{a} \\
\delta_{e} \\
\delta_{r}
\end{array}\right]
$$

where $\chi_{r}(u, v, w, p, q, r) \in \mathbb{R}^{3 \times 1}, \gamma_{r}(u, v, w) \in \mathbb{R}^{3 \times 3}$. It is important to remark that matrix $\gamma_{r}(u, v, w)$ is invertible in the required flight envelop.

The derivatives of (7) can be found easily using (9):

$$
\left[\begin{array}{c}
\dot{\zeta}_{10} \\
\dot{\zeta}_{11} \\
\dot{\zeta}_{12} \\
\dot{\sigma}_{2}
\end{array}\right]=\chi_{T}\left(\tilde{X}, p, q, r, v_{4}\right)+\gamma_{1}(\tilde{X})\left[\begin{array}{cc}
\gamma_{r}(u, v, w) 0 \\
0 & 1
\end{array}\right]\left[\begin{array}{l}
\delta_{a} \\
\delta_{e} \\
\delta_{r} \\
\dot{v}_{4}
\end{array}\right]
$$

where

$$
\chi_{T}(\tilde{X})=\dot{\chi}_{1}(\tilde{X})+\dot{\gamma}_{1}(\tilde{X})\left[\begin{array}{c}
p \\
q \\
r \\
v_{4}
\end{array}\right]+\gamma_{1}(\tilde{X})\left[\begin{array}{c}
\chi_{r}(\cdot) \\
0
\end{array}\right]
$$

By defining $v_{4}=\tau$ as a state variable, $\dot{v}_{4}$ as the input $u_{T}$, we have a feedback linearizable system in (5) and (10) with 14 states by the change of coordinates from $(x, y, z, u, v, w, \phi, \theta, \psi, p, q, r, \eta, \tau)$ to $\left(\zeta_{1}, \zeta_{2}, \zeta_{3}, \zeta_{4}\right.$, $\left.\zeta_{5}, \zeta_{6}, \zeta_{7}, \zeta_{8}, \zeta_{9}, \zeta_{10}, \zeta_{11}, \zeta_{12}, \sigma_{1}, \sigma_{2}\right)$

In system (5) and (10), we have 12 physical states from the aircraft, and 2 states from the integration of thrust. We need now the nonsingularity of matrix:

$$
\gamma_{T}(\cdot)=\gamma_{1}(\tilde{X})\left[\begin{array}{cc}
\gamma_{r}(u, v, w) & 0 \\
0 & 1
\end{array}\right]
$$

Now we will define $\sigma_{1}$ as $\phi$, which has the dynamics:

$$
\dot{\phi}=p+q \tan \theta s \phi+r \tan \theta c \phi
$$

Matrix $\gamma_{1}(\cdot)$ then becomes

$$
\gamma_{1}(\cdot)=\left[\begin{array}{llll}
D_{1}^{1} & D_{1}^{2} & D_{1}^{3} & D_{1}^{4} \\
D_{2}^{1} & D_{2}^{2} & D_{2}^{3} & D_{2}^{4} \\
D_{3}^{1} & D_{3}^{2} & D_{3}^{3} & D_{3}^{4} \\
1 & \tan \theta s \phi & \tan \theta c \phi & 0
\end{array}\right]
$$

The nonsingularity of matrix $\gamma_{1}(\cdot)$ in this case is guaranteed in the required flight envelop. It is physically explained that three control variables are used to control the trajectory 
of the aircraft, and the last control variable to control the roll motion. The linearizing feedback is given as:

$$
\begin{aligned}
& {\left[\begin{array}{c}
\delta_{a} \\
\delta_{e} \\
\delta_{r} \\
u_{T}
\end{array}\right]=\gamma_{T}^{-1}(\cdot)\left(-\chi_{T}(\cdot)+\left[\begin{array}{c}
\dot{\zeta}_{10 r} \\
\dot{\zeta}_{11 r} \\
\dot{\zeta}_{12 r} \\
\dot{\sigma}_{2 r}
\end{array}\right]+\left[\begin{array}{c}
-k_{11}\left(\zeta_{1}-\zeta_{1 r}\right) \\
-k_{21}\left(\zeta_{2}-\zeta_{2 r}\right) \\
-k_{31}\left(\zeta_{3}-\zeta_{3 r}\right) \\
0
\end{array}\right.\right.} \\
& -k_{12}\left(\zeta_{4}-\zeta_{4 r}\right)-k_{13}\left(\zeta_{7}-\zeta_{7 r}\right)-k_{14}\left(\zeta_{10}-\zeta_{10 r}\right) \\
& -k_{22}\left(\zeta_{5}-\zeta_{5 r}\right)-k_{23}\left(\zeta_{8}-\zeta_{8 r}\right)-k_{24}\left(\zeta_{11}-\zeta_{11 r}\right) \\
& \left.\begin{array}{r}
-k_{32}\left(\zeta_{6}-\zeta_{6 r}\right)-k_{33}\left(\zeta_{9}-\zeta_{9 r}\right)-k_{34}\left(\zeta_{12}-\zeta_{12 r}\right) \\
0-k_{33}\left(\sigma_{1}-\sigma_{1 r}\right)-k_{44}\left(\sigma_{2}-\sigma_{2 r}\right)
\end{array}\right]
\end{aligned}
$$

where the $k_{i j}$ for $1 \leq i, j \leq 4$ are positive parameters to be tunned, the output references are defined as:

$$
\left\{\begin{array}{l}
\left(x_{r}, y_{r}, z_{r}\right)^{T}=\left(\zeta_{1 r}, \zeta_{2 r}, \zeta_{3 r}\right)^{T}=R_{1} \\
\left(\dot{x}_{r}, \dot{y}_{r}, \dot{z}_{r}\right)^{T}=\left(\zeta_{4 r}, \zeta_{5 r}, \zeta_{6 r}\right)^{T}=R_{2} \\
\left(\ddot{x}_{r}, \ddot{y}_{r}, \ddot{z}_{r}, \phi_{r}\right)^{T}=\left(\zeta_{7 r}, \zeta_{8 r}, \zeta_{9 r}, \sigma_{1 r}\right)^{T}=R_{3} \\
\left(x_{r}^{(3)}, y_{r}^{(3)}, z_{r}^{(3)}, \dot{\phi}_{r}\right)^{T}=\left(\zeta_{10 r}, \zeta_{11 r}, \zeta_{12 r}, \sigma_{2 r}\right)^{T}=R_{4} \\
\left(x_{r}^{(4)}, y_{r}^{(4)}, z_{r}^{(4)}, \ddot{\phi}_{r}\right)^{T}=\left(\dot{\zeta}_{10 r}, \dot{\zeta}_{11 r}, \dot{\zeta}_{12 r}, \dot{\sigma}_{2 r}\right)^{T}=R_{5}
\end{array}\right.
$$

\section{Stability Analysis}

In the following, we demonstrate that an aircraft is exponentially stabilized by the previously designed controller in considering the effect of moments and control surfaces on the aerodynamic force. We suppose that the effect of moment is defined by function $\epsilon_{1} \Psi_{1}(X)$, the effect of control surfaces is defined by function $\epsilon_{2} \Psi_{2}(X) u_{a}(X)$ where $X=$ $(x, y, z, u, v, w, \phi, \theta, \psi, p, q, r, \eta, \tau)^{T}$. This system is called slightly non - minimum phase system, a stability analysis for such systems can be seen in [13].

We define state vectors,

$$
\left\{\begin{array}{l}
\xi_{1}=(x, y, z)^{T}=\left(\zeta_{1}, \zeta_{2}, \zeta_{3}\right)^{T} \\
\xi_{2}=\dot{\xi_{1}}=(\dot{x}, \dot{y}, \dot{z})^{T}=\left(\zeta_{4}, \zeta_{5}, \zeta_{6}\right)^{T} \\
\xi_{3}=\dot{\xi_{2}}=(\ddot{x}, \ddot{y}, \ddot{z})^{T}=\left(\zeta_{7}, \zeta_{8}, \zeta_{9}\right)^{T} \\
\xi_{4}=\dot{\xi_{3}}=\left(x^{(3)}, y^{(3)}, z^{(3)}\right)^{T}=\left(\zeta_{10}, \zeta_{11}, \zeta_{12}\right)^{T} \\
\xi_{1 \phi}=\phi=\sigma_{1} ; \xi_{2 \phi}=\dot{\xi}_{1 \phi}=\dot{\phi}=\sigma_{2}
\end{array}\right.
$$

From (5), (10) and (16), the approximate system can be described as:

$$
\left\{\begin{array}{l}
\dot{\xi}_{1}=\xi_{2} \\
\dot{\xi}_{2}=\xi_{3} \\
\dot{\xi}_{3}=\xi_{4} \\
\dot{\xi}_{1 \phi}=\xi_{2 \phi} \\
\dot{\xi}_{4}=\chi_{T 0}(X)+\gamma_{T 0}(X) u_{a} \\
\dot{\xi}_{2 \phi}=\chi_{T 1}(X)+\gamma_{T 1}(X) u_{a}
\end{array}\right.
$$

where the matrix $\gamma_{T}(X)=\left[\begin{array}{c}\gamma_{T 0}(X) \\ \gamma_{T 1}(X)\end{array}\right]$ is nonsingular in the studied field of $X$ and $\chi_{T}(X)=\left[\begin{array}{c}\chi_{T 0}(X) \\ \chi_{T 1}(X)\end{array}\right], \gamma_{T 0}(X) \in$ $\mathbb{R}^{3 \times 4}, \gamma_{T 1}(X) \in \mathbb{R}^{1 \times 4}, \chi_{T 0}(X) \in \mathbb{R}^{3 \times 1}$ and $\chi_{T 0}(X) \in \mathbb{R}$.

Computing the effects of moment and of control surfaces, the true system can be described as:

$$
\left\{\begin{array}{l}
\dot{\xi}_{1}=\xi_{2} \\
\dot{\xi}_{2}=\xi_{3}+\frac{\partial \xi_{2}}{\partial x}\left(\epsilon_{1} \psi_{1}(X)+\epsilon_{2} \psi_{2}(X) u_{a}\right) \\
\dot{\xi}_{3}=\xi_{4}+\frac{\partial \xi_{3}}{\partial x}\left(\epsilon_{1} \psi_{2}(X)+\epsilon_{2} \psi_{2}(X) u_{a}\right) \\
\dot{\xi}_{1 \phi}=\xi_{2 \phi} \\
\dot{\xi}_{4}=\chi_{T 0}(X)+\gamma_{T 0}(X) u_{a}+\frac{\partial \xi_{4}}{\partial x}\left(\epsilon_{1} \psi_{1}(X)+\epsilon_{2} \psi_{2}(X) u_{a}\right) \\
\dot{\xi}_{2}=\chi_{T}(X)+\gamma_{T}(X) u_{a}
\end{array}\right.
$$

The approximate tracking controller that we designed in the last subsection is:

$$
\begin{aligned}
u_{a}= & {\left[\begin{array}{c}
\gamma_{T 0}(X) \\
\gamma_{T 1}(X)
\end{array}\right]^{-1}\left(-\left[\begin{array}{c}
\chi_{T 0}(X) \\
\chi_{T 1}(X)
\end{array}\right]-K_{1}\left(\xi_{1}-R_{1}\right)-K_{2}\left(\xi_{2}-R_{2}\right)\right.} \\
& \left.-K_{3}\left(\left[\begin{array}{c}
\xi_{3} \\
\xi_{1 \phi}
\end{array}\right]-R_{3}\right)-K_{4}\left(\left[\begin{array}{c}
\xi_{4} \\
\xi_{2 \phi}
\end{array}\right]-R_{4}\right)+R_{5}\right)
\end{aligned}
$$

where $K_{3}=\operatorname{diag}\left(k_{13}, k_{23}, k_{33}, k_{43}\right), \quad K_{4}=$ $\operatorname{diag}\left(k_{14}, k_{24}, k_{34}, k_{44}\right)$ and

$$
K_{1}=\left[\begin{array}{ccc}
k_{11} & 0 & 0 \\
0 & k_{21} & 0 \\
0 & 0 & k_{31} \\
0 & 0 & 0
\end{array}\right] ; K_{2}=\left[\begin{array}{ccc}
k_{12} & 0 & 0 \\
0 & k_{22} & 0 \\
0 & 0 & k_{32} \\
0 & 0 & 0
\end{array}\right]
$$

We define the trajectory error vector $e_{1}=\xi_{1}-R_{1}, e_{2}=$ $\xi_{2}-R_{2},\left[\begin{array}{c}e_{3} \\ e_{5}\end{array}\right]=\left[\begin{array}{c}\xi_{3} \\ \xi_{1 \phi}\end{array}\right]-R_{3}$ and $\left[\begin{array}{c}e_{4} \\ e_{6}\end{array}\right]=\left[\begin{array}{c}\xi_{4} \\ \xi_{2 \phi}\end{array}\right]-R_{4}$.

Then the system (18) with the approximate tracking controller in (14) can be rewritten as:

$$
\begin{aligned}
& \left.\left.\left[\begin{array}{c}
\dot{e}_{1} \\
\dot{e_{2}} \\
\dot{e_{3}} \\
\dot{e_{5}} \\
\dot{e_{4}} \\
\dot{e_{6}}
\end{array}\right]\right]=\left[\begin{array}{cccc}
I_{3} & 0 & 0 & 0 \\
0 & I_{3} & 0 & 0 \\
0 & 0 & I_{4} & 0 \\
-K_{1}-K_{2} & -K_{3} & -K_{4}
\end{array}\right]\left[\begin{array}{c}
e_{1} \\
e_{2} \\
e_{3} \\
e_{5} \\
e_{4} \\
e_{6}
\end{array}\right]\right] \\
& +\left[\begin{array}{c}
0 \\
\frac{\partial \xi_{2}}{\partial X} \epsilon_{1} \psi_{1}(X) \\
\frac{\partial \xi_{3}}{\partial X} \epsilon_{1} \psi_{1}(X) \\
0 \\
\frac{\partial \xi_{4}}{\partial X} \epsilon_{1} \psi_{1}(X) \\
0
\end{array}\right]+\left[\begin{array}{c}
0 \\
\frac{\partial \xi_{2}}{\partial X} \epsilon_{2} \psi_{2}(X) u_{a}(X) \\
\frac{\partial \xi_{3}}{\partial X} \epsilon_{2} \psi_{2}(X) u_{a}(X) \\
0 \\
\frac{\partial \xi_{4}}{\partial X} \epsilon_{2} \psi_{2}(X) u_{a}(X) \\
0
\end{array}\right]
\end{aligned}
$$

Or compactly,

$$
\dot{e}=A e+\epsilon_{1} \Psi_{1}(X)+\epsilon_{2} \Psi_{2}(X) u_{a}(X)
$$

We will show now that $e$ is bounded. To this end, we consider the Lyapunov candidate:

$$
W=e^{T} P e
$$

where matrix $P$ is the solution of:

$$
A^{T} P+P A=-I_{14}
$$

By the assumption that all output references are bounded, that means $R_{i}$ for $1 \leq i \leq 5$ are bounded, we have:

$$
\|\xi\| \leq\|e\|+b_{d}
$$

Furthermore, we can check that $\Psi_{1}(X)$ and $\Psi_{2}(X) u_{a}(X)$ are locally Lipschitz, note that $X$ is a local diffeomorphism of $\xi$, so $\|X\| \leq l_{x}\|\xi\|$.

$$
\begin{gathered}
\left\|P \Psi_{1}(X)\right\| \leq l_{1}\|X\| \leq l_{1} l_{x}\|\xi\| \\
\left\|P \Psi_{2}(X) u_{a}(X)\right\| \leq l_{2}\|X\| \leq l_{2} l_{x}\|\xi\|
\end{gathered}
$$

Take the derivative of Lyapunov function, we find that:

$$
\begin{aligned}
\dot{W}= & -e^{T} e+2 e^{T} P\left(\epsilon_{1} \Psi_{1}(X)+\epsilon_{2} \Psi_{2}(X) u_{a}(X)\right) \\
\leq & -\|e\|^{2}+2\|e\|\left(\epsilon_{1} l_{1} l_{x}\|\xi\|+\epsilon_{2} l_{2} l_{x}\|\xi\|\right) \\
\leq & -\|e\|^{2}+2\|e\|\left(\epsilon_{1} l_{1} l_{x}\left(\|e\|+b_{d}\right)+\epsilon_{2} l_{2} l_{x}\left(\|e\|+b_{d}\right)\right) \\
\leq & -\|e\|^{2}+2\left(\epsilon_{1} l_{1} l_{x}+\epsilon_{2} l_{2} l_{x}\right)\|e\|^{2}+2\left(\epsilon_{1} l_{1} l_{x}\right. \\
& \left.+\epsilon_{2} l_{2} l_{x}\right) b_{d}\|e\| \\
\leq & -\left(3 / 4-2\left(\epsilon_{1} l_{1} l_{x}+\epsilon_{2} l_{2} l_{x}\right)\right)\|e\|^{2}-\left(\|e\| / 2-2\left(\epsilon_{1} l_{1} l_{x}\right.\right. \\
& \left.\left.+\epsilon_{2} l_{2} l_{x}\right) b_{d}\right)^{2}+\left(2\left(\epsilon_{1} l_{1} l_{x}+\epsilon_{2} l_{2} l_{x}\right) b_{d}\right)^{2}
\end{aligned}
$$

Thus $\dot{W} \leq 0$ whenever $e$ is large, then $\xi$ and $X$ are bounded. If we choose $b_{d}$ sufficiently small and with an approximate initial conditions, all system states remain in a small neighborhood of tracking values. For the purpose of stabilization of aircraft system after the launching phase, the references and its derivatives are zero, $b_{d}$ is zero. The system is exponentially stable with $\epsilon_{1}$ and $\epsilon_{2}$ are sufficiently small. We can see that for the purpose of flight stabilization, dynamic feedback linearization controller satisfies the performance requirement. 


\section{Simulation Results}

This section will present numerical simulation results of the proposed feedback linearization control laws designed in section III to demonstrate the performance in the drop phase.

As mentioned in section II, we assume that the launch phase is considered as a perturbation on aerodynamic force and moment during an interval $T_{i n t}$ that affect the aircraft model following the launch phase, that is taken as an F16. This model is used since this aircraft has already been applied for (manned) airlaunch, and because its nonlinear model, wind tunnel informations and data are widely known and used for control design. It is important to remark that the model used in the following simulations is even more complete than that used in the control design, for example it includes actuator dynamics and their limitations. As a consequence, simulations also illustrate some properties of robustness to unmodeled dynamics. We may note that the control inputs are bounded by their physical limitations introduced in section II.

In the following text, we will show simulations of the stabilization of the aircraft model following the launch phase to its equilibrium point $(V, h)=(154 m / s, 6500 m)$ corresponding to (angle of attack $\alpha_{r}$ to $2.7^{\circ}$, sideslip $\beta_{r}$ to $0^{\circ}$, and roll angle $\phi_{r}$ to $0^{\circ}$ ).
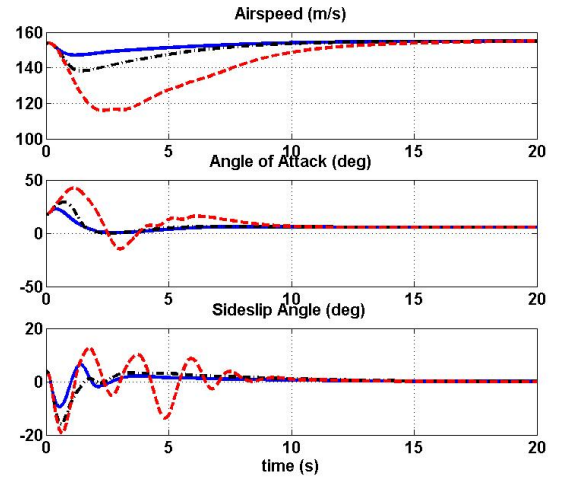

Fig. 1. Airspeed, Angle of attack and sideslip angle

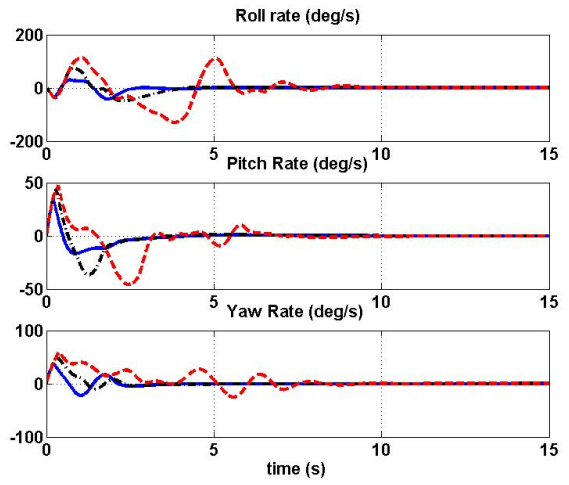

Fig. 2. State variables: Angular rates of system

Its initial condition is the final state of the first model (before the drop phase $-\alpha=12.5^{\circ}, \beta=0^{\circ}$ and $\phi=0^{\circ}$ ) as in section II. Moreover, we add on its initial condition a small disturbance on system output. That means the initial condition of the second model is $\left(\alpha_{0}=17.5^{\circ}, \beta_{0}=4^{\circ}\right.$ and $\phi_{0}=10^{\circ}$ ) for all numerical simulations.
The second model is disturbed on aerodynamic force and moment during an interval $T_{i n t}$ as in section II. We simulate three sets of $T_{\text {int }}$,

1) $T_{\text {int }}=0.2 \mathrm{~s}$ (solid lines in Fig. 1 to Fig. 5),

2) $T_{\text {int }}=0.3 \mathrm{~s}$ (dashed lines in Fig. 1 to Fig. 5)

3) $T_{\text {int }}=0.36 \mathrm{~s}$ (dash dotted lines in Fig. 1 to Fig. 5)

It is interesting to remind that the aircraft with constant inputs (trim conditions) is unstable for $T_{\text {int }}$ greater than $0.227 \mathrm{~s}$ (see [15]). The dynamic feedback linearization controller we designed in section III will stabilize the system for longer periods. Finally, we note that the system will be unstable for an interval $T_{\text {int }}$ greater than $0.36 \mathrm{~s}$. Nevertheless, in this case the control inputs are strongly saturated. As a consequence this time interval represents more likely the limitations of the aircraft itself than the limitations of the control algorithm.

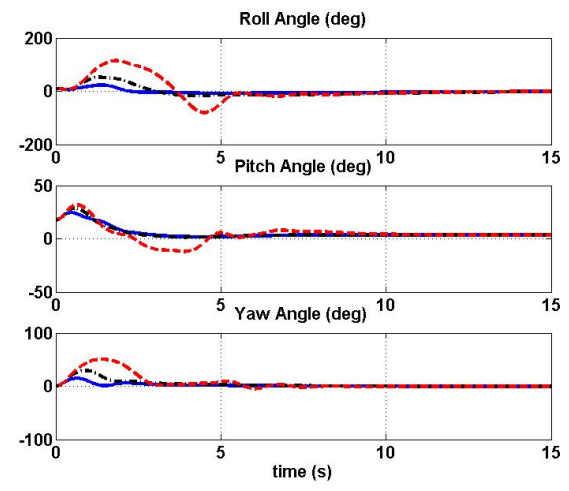

Fig. 3. State variables: Euler's angle of system

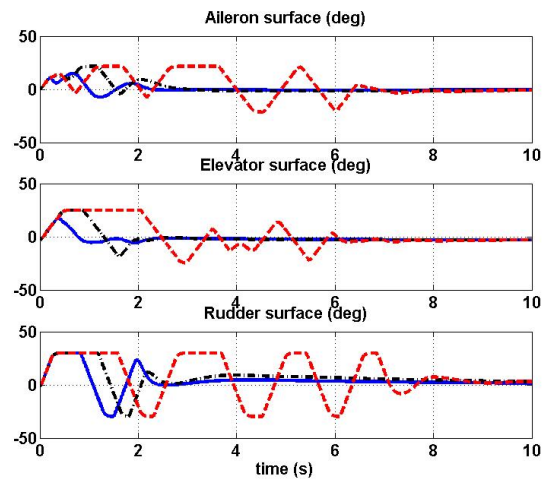

Fig. 4. Control surface: aileron, elevator and Rudder

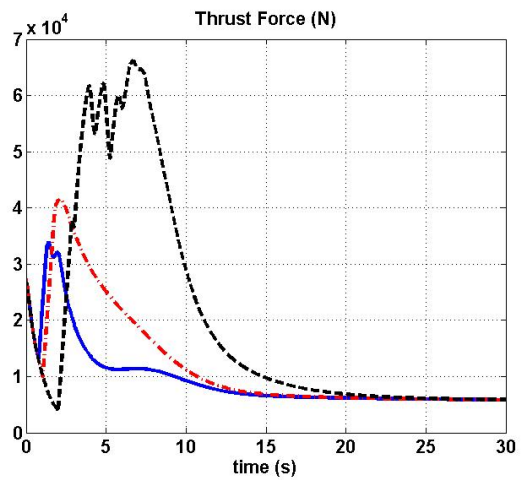

Fig. 5. Thrust Force

Figs. 1 to 3 represent the convergence of the system states to the operating point of the aircraft at the end of $10 \mathrm{~s}$ for three cases of $T_{\text {int }}=(0.2 s, 0.3 s, 0.36 s)$. For $T_{\text {int }}=(0.2 s, 0.3 s)$ the system is well stabilized, while for $T_{\text {int }}$ assuming larger 
values the system becomes more oscillatory and attains its limits of stability in case of $T_{i n t}=0.36 \mathrm{~s}$.

Fig. 4 shows how the control variables and thrust behave for the three cases of study. The control surfaces in the last case are saturated by their physical limitations due to a high perturbation on aerodynamic force and moment. It can be said that the performance of the Feedback Linearization Controller allows the system to keep stability even with large perturbation during a long time interval $T_{\text {int }}$.

\section{Collision Avoidance}

Airlaunch problem does not only require stability of system's states, but also to avoid the possibility of collision between the aircraft and the rocket after the drop phase. Fig. 6 shows the altitude of the aircraft from 0 to $1 s$ in the three previous cases of study by using the Feedback Linearization Controller. They are compared with the trajectory of the rocket that drop freely with the initial airspeed of the aircraft (the thin solid plot). It is important to remark that there is a small distance from the initial height of the rocket and the aircraft, representing the distance from the respective centers of mass.

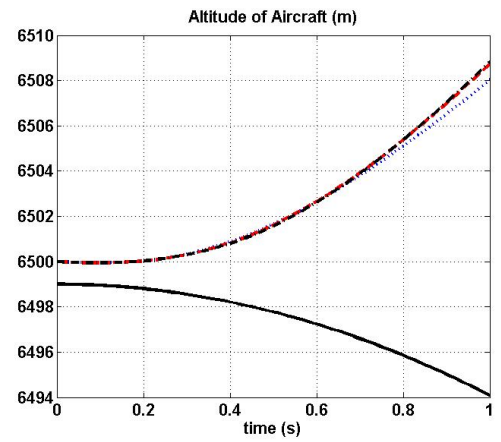

Fig. 6. Altitude of the aircraft with Feedback Linearization Controller

In the three cases (dotted plot, dashed plot and dash dotted plot), the altitude of the aircraft satisfies the specification that requires there is no collision between the aircraft and the rocket in the airlaunch phase.

\section{CONCLUSION}

We have presented the design of a Dynamic Feedback Linearization Controller aiming to stabilize an unmanned aircraft performing the airlaunch of a satellite launcher (rocket). The paper shows that the flight system can be dynamic linearizable (using a second order integration of the thrust input) in a first step where it was neglected the derivatives of aerodynamic forces with respect to control surfaces and angular rates. In a second step, a deeper analysis shows that this dynamic feedback linearization controller stabilizes the aircraft even when are taken into account the effects of the control surfaces and angular rates on the aerodynamic forces.

The proposed controller is then applied to an F-16 model disturbed by large impulses on forces and moments that may destabilize the system. This represents an aircraft performing airlaunch, just after dropping the second stage. The performance of the proposed controller is illustrated by computer simulations with initial conditions representing the final (stable) state before the launch phase. In three of the studied cases, the stability of the system after the drop phase is assured, all states return to their equilibrium values, and there is no collision between the aircraft and the rocket, even if the the perturbation interval $T_{\text {int }}$ becomes large. The controller satisfies then the requirement of flight stabilization and collision avoidance with the rocket.

\section{REFERENCES}

[1] B. Charlet, J. Levine, and R. Marino. On dynamic feedback linearization. Systems \& Control Letters, 13:143-151, 1989.

[2] B. Charlet, J. Levine, and R. Marino. Dynamic feedback linearization with application to aircraft control. In Proceedings of the 27th Conference on Decision and Control Austin, Texas, USA, 1:701-705, December 7 - 9, 1988.

[3] B. Charlet, J. Levine, and Riccardo Marino. New sufficient conditions for dynamic feedback linearization. IFAC Symposium Capri, Italy, June 14-16, 1989.

[4] B. Charlet, J. Levine, and Marino R. Sufficient conditions for dynamic feedback linearization. SIAM Journal of Control and Optimization, 1991.

[5] Gilney Damm and Van Cuong Nguyen. Mimo conditional integrator control for a class of nonlinear systems. In In proceedings of System Theory, Control, and Computing (ICSTCC), 2011 15th International Conference, Sinaia, Romania, October 14-16, 2011.

[6] John Hauser, Shankar Sastry, and George Meyer. Nonlinear control design for slightly non-minimum phase systems: Application to v/stol aircraft. Automatica, 28:665-679, July, 1992.

[7] Gary C. Hudson. Quickreach responsive launch system. In 4th Responsive Space Conference, Los Angeles, CA, USA, April 24-27, 2006.

[8] L. R. Hunt, Renjeng Su, and George Meyer. Global transformations of nonlinear systems. IEEE Transactions on Automatic Control, 28:2431, January, 1983.

[9] Stevens Brian L. and Lewis Frank L. Aircraft Control and Simulation. John Wiley \& Sons, 1992.

[10] Stephen H. Lane and Robert F. Stengel. Flight control design using non-linear inverse dynamics. American Control Conference, Seattle, WA, USA, pages 587-596, June 18-20, 1986.

[11] Taeyoung Lee and Youdan Kim. Nonlinear adaptive flight control using backstepping and neural networks controller. Journal of guidance, control and dynamics, 24:675-682, 2001.

[12] Attaullah Y. Memona and Hassan K. Khalil. Output regulation of nonlinear systems using conditional servocompensators. Automatica, 46:1119-1128, July, 2010.

[13] G. Meyer, R. Su, and L. R. Hunt. Application of nonlinear transformations to automatic flight control. Automatica, 20:103 - 107, January, 1984.

[14] L.T. Nguyen, M.E. Ogburn, and P. Deal. Simulator study of fighter airplane with relaxed longitudinal static stability. Technical report NASA, page 1538, 1979.

[15] Van Cuong Nguyen and Gilney Damm. Modeling and conditional integrator control of an unmanned aerial vehicle for airlaunch. in Proceedings of the 2012 American Control Conference (ACC), Montreal, Canada, June 27-29, 2012.

[16] Van Cuong Nguyen and Gilney Damm. Mimo conditional integrator control for unmanned airlaunch. In In proceedings of System Theory, Control, and Computing (ICSTCC), 2011 15th International Conference, Sinaia, Romania, October 14-16, 2011.

[17] H. Nijmeijer and A.J. Van der Schaft. Nonlinear dynamical control systems. Springer, 1990.

[18] John W.C. Robinson. Block backstepping for nonlinear flight control law design. Springer, pages 231-257, 2007.

[19] Jan Roskam. Airplane Flight Dynamic and Automatic Flight Controls, Part 1. Design, Analysis and Research Corporation, 2001.

[20] Marti Sarigul-Klijn and Nesrin Sarigul-Klijn. Selection of a carrier aircraft and a launch method for air launching space vehicles. In $A I A A$ SPACE 2008 Conference and Exposition, San Diego, California, USA, September 9 - 11, 2008.

[21] Qian Wang and Robert F. Stengel. Robust nonlinear flight control of a high-performance aircraft. IEEE Transaction on control systems technology, 13:15-26, 2005. 